\title{
BMJ Open Biological rationale and potential clinical use of gabapentin and pregabalin in bipolar disorder, insomnia and anxiety: protocol for a systematic review and meta-analysis
}

\author{
Kerensa T Houghton, ${ }^{1}$ Alexandra Forrest, ${ }^{1,2}$ Amine Awad, ${ }^{1}$ Lauren Z Atkinson, ${ }^{1}$ \\ Sarah Stockton, ${ }^{1}$ Paul J Harrison, ${ }^{1,2}$ John R Geddes, ${ }^{1,2}$ Andrea Cipriani ${ }^{1,2}$
}

To cite: Houghton $\mathrm{KT}$, Forrest A, Awad A, et al. Biological rationale and potential clinical use of gabapentin and pregabalin in bipolar disorder, insomnia and anxiety: protocol for a systematic review and metaanalysis. BMJ Open 2017;7: e013433. doi:10.1136/ bmjopen-2016-013433

- Prepublication history for this paper is available online. To view these files please visit the journal online (http://dx.doi.org/10.1136/ bmjopen-2016-013433).

Received 12 July 2016 Revised 6 January 2017 Accepted 13 January 2017

CrossMark

\section{${ }^{1}$ Department of Psychiatry, University of Oxford, Warneford Hospital, Oxford OX3 7JX, UK \\ ${ }^{2}$ Oxford Health NHS \\ Foundation Trust, Warneford Hospital, Oxford OX3 7JX, UK}

Correspondence to Professor Andrea Cipriani; andrea.cipriani@psych.ox.ac.uk

\section{ABSTRACT}

Introduction: Gabapentin has been extensively prescribed off-label for psychiatric indications, with little established evidence of efficacy. Gabapentin and pregabalin, a very similar drug with the same mechanism of action, bind to a subunit of voltagedependent calcium channels which are implicated in the aetiopathogenesis of bipolar disorder, anxiety and insomnia. This systematic review and meta-analysis aims to collect and critically appraise all the available evidence about the efficacy and tolerability of gabapentin and pregabalin in the treatment of bipolar disorder, insomnia and anxiety.

Methods and analysis: We will include all randomised controlled trials (RCTs) reported as double-blind and comparing gabapentin or pregabalin with placebo or any other active pharmacological treatment (any preparation, dose, frequency, route of delivery or setting) in patients with bipolar disorder, anxiety or insomnia. For consideration of adverse effects (tolerability), single-blind or open-label RCTs and non-randomised evidence will also be summarised. The main outcomes will be efficacy (measured as dichotomous and continuous outcome) and acceptability (proportion of patients who dropped out of the allocated treatment). Published and unpublished studies will be sought through relevant database searches, trial registries and websites; all reference selection and data extraction will be conducted by at least 2 independent reviewers. We will conduct a random-effects meta-analysis to synthesise all evidence for each outcome.

Heterogeneity between studies will be investigated by the $\mathrm{I}^{2}$ statistic. Data from included studies will be entered into a funnel plot for investigation of smallstudy effects. No subgroup analysis will be undertaken, but we will carry out sensitivity analyses about combination treatment, psychiatric comorbidity, use of rescue medications and fixed versus randomeffects model.

Ethics and dissemination: This review does not require ethical approval. This protocol has been registered on PROSPERO (CRD42016041802).

\section{Strengths and limitations of this study}

- Gabapentin and pregabalin have been extensively prescribed off-label for psychiatric indications, including bipolar disorder, sleep and anxiety, with little established evidence of efficacy. We will conduct a systematic review of all available published and unpublished literature and will carry out a random-effects pairwise meta-analysis to synthesise all available evidence for each outcome, if possible.

- To assess efficacy and acceptability, only doubleblind, randomised controlled trials will be included, while, for consideration of adverse effects (tolerability), single-blind or open-label trials and non-randomised evidence will also be summarised.

- Gabapentin and pregabalin are ligands of the $\alpha 2 \delta$ subunit of voltage-dependent calcium channels, which are currently under investigation as a target for novel pharmaceuticals to be used in the management of bipolar disorder.

- The risk of publication bias and the risk of selection bias are high in the psychiatric literature, in particular with gabapentin.

- The limitations of primary studies will be addressed with the Cochrane risk of bias tool and the quality of evidence contributing to pooled estimates will be assessed with the GRADE framework.

The results of the systematic review will be disseminated via publication in a peer-reviewed journal.

\section{BACKGROUND}

Gabapentin was first licenced by the Food and Drug Administration (FDA) in 1993 as an adjunctive treatment for partial seizures in people aged over 12 years. ${ }^{1}$ It was 
subsequently discovered to have analgesic properties ${ }^{2-4}$ and licenced by the FDA for use in postherpetic neuralgia in 2004. In the same year, pregabalin, a structural analogue of gabapentin, was licenced for the treatment of neuropathic pain and as an adjunct in the management of epilepsy. ${ }^{5-7}$ Pregabalin has since been licenced to treat generalised anxiety disorder. ${ }^{8}$ Gabapentin has a long history of off-label prescription. ${ }^{9}$ Between 1998 and 2000, a study involving 105 Medicaid patients who were prescribed gabapentin found that $95 \%$ of prescriptions had been for off-label indications. ${ }^{10}$ A number of these indications were for psychiatric illness, including bipolar disorder (10\% of all prescriptions in the study) and anxiety disorders. Despite the prevalent prescription of gabapentin for psychiatric illness spanning over 20 years, there has been limited investigation into its efficacy for such disorders. Berlin et $a l^{11}$ conducted a systematic review of the use of gabapentin in psychiatric illness, but did not search unpublished trials. They reported mixed evidence as to the efficacy of gabapentin in bipolar disorder. One randomised controlled trial (RCT) comparing adjunctive use of gabapentin in bipolar mania or depression with placebo found no improvement of symptoms in the gabapentin group. ${ }^{12}$ Another two studies, which compared gabapentin monotherapy in refractory bipolar disorder with placebo and lamotrigine, found gabapentin to be ineffective in treating mania or depression. ${ }^{13}{ }^{14}$ Additionally, a meta-analysis conducted to compare treatments of acute mania found gabapentin to be less efficacious than placebo. ${ }^{15}$ However, a trial which compared gabapentin with placebo in the maintenance of bipolar disorder concluded that gabapentin may provide some benefit in the long-term treatment of bipolar disorder. ${ }^{16}$ The evidence for gabapentin in anxiety disorder is similarly incomplete. Only one RCT has been completed to compare gabapentin and placebo as adjunctive therapy in social phobia, suggesting that gabapentin is more effective than placebo. ${ }^{17}$ Other trials of gabapentin in anxiety have focused on preoperative anxiety and have mostly found gabapentin to be more effective than placebo. ${ }^{11}$

Given the lack of evidence of efficacy of gabapentin in bipolar disorder and anxiety, it may be difficult to understand the widespread prescription of the drug for these off-licence indications. However, the pharmaceutical company which promoted gabapentin, Pfizer, has been fined in the USA for illegal promotion of gabapentin for unlicenced indications. ${ }^{18}$ It is therefore uncertain whether the historical widespread prescription of gabapentin for psychiatric illness was due to efficacy noted by individual physicians and patients, or due to illegal promotion of the drug for off-label use.

Pregabalin has been found to be effective in the management of generalised anxiety disorder, ${ }^{19}$ and is licenced in the UK for this indication. There is also some evidence, from an open-label observational study, that pregabalin is effective in the adjunctive treatment of acute mania, depression, and maintenance of refractory bipolar disorder. ${ }^{20}$ Gabapentin was originally designed to be structurally similar to the neurotransmitter $\gamma$-aminobutyric acid (GABA) ${ }^{21}$ It was intended as an antispasmodic drug which would act on GABA receptors and have GABAergic properties. ${ }^{22}$ In fact, neither gabapentin nor pregabalin bind to GABA receptors. Both drugs are ligands of the $\alpha 2 \delta$ subunit of voltagedependent calcium channels, ${ }^{2324}$ and there is evidence that their antiepileptic and analgesic properties are directly related to their calcium channel interaction. ${ }^{25} 26$ Voltage-dependent calcium channels are currently under investigation as a target for novel pharmaceuticals to be used in the management of bipolar disorder, and they have four subunits:

- $\alpha 1$ subunit, which forms the transmembrane calcium-selective pore $;^{27}$

- $\beta$ and $\alpha 2 \delta$ subunits, which are involved in trafficking of the $\alpha 1$ subunit to the cell membrane; ${ }^{28}$

- $\gamma$ subunit, which is not involved in trafficking, but appears to influence biophysical properties of associated calcium channels. ${ }^{29}$

Voltage-gated calcium channels are classified into three main groups depending on sequence homology of the $\alpha 1$ subunit: ${ }^{30}$ (1) $\mathrm{Ca}_{\mathrm{v}} 1$, or L-type channels; (2) $\mathrm{Ca}_{\mathrm{v}} 2$, of which there are four subtypes known as $\mathrm{P}, \mathrm{Q}, \mathrm{N}$ and R-type channels; (3) $\mathrm{Ca}_{\mathrm{v}}$ 3, also called T-type calcium channels. L-type calcium channels $\left(\mathrm{Ca}_{\mathrm{v}} 1\right)$ may be further classified into types $\mathrm{Ca}_{\mathrm{v}} 1.1, \mathrm{Ca}_{\mathrm{v}} 1.2, \mathrm{Ca}_{\mathrm{v}} 1.3$ and $\mathrm{Ca}_{\mathrm{v}}$ 1.4. The channel $\mathrm{Ca}_{\mathrm{v}} 1.2$, which is found in skeletal muscle, heart and brain, is coded for by the gene CACNA1C. Genome-wide association studies have consistently found an association between a common singlenucleotide polymorphism in the CACNA1C gene and bipolar disorder, ${ }^{31}{ }^{32}$ which appears likely to increase $\mathrm{Ca}_{\mathrm{v}} 1.2$ channel expression and function. ${ }^{33} 34$ Other evidence that calcium channels may be involved in the aetiopathogenesis of bipolar disorder is that agonist-stimulated calcium response, which is a key pathway in intracellular secondary messaging, is enhanced in the platelets of patients with bipolar disorder. ${ }^{35}$ Additionally, mitochondria influence the sequestration of excess intracellular calcium which accumulates as a result of agonist stimulation and magnetic resonance studies have found mitochondrial dysfunction in patients with bipolar disorder. $^{36} 37$

This evidence suggests that calcium channels, particularly $\mathrm{Ca}_{\mathrm{v}} 1.2$ channels, may be a useful target in the design of novel therapies for bipolar disorder. Further, since the genetic mutations associated with bipolar disorder are likely to increase $\mathrm{Ca}_{\mathrm{v}} 1.2$ expression and function, drugs which act as antagonists to the action of this channel may be found to be effective in bipolar disorder. A recent review found no evidence that verapamil, an L-type calcium channel blocker usually prescribed for cardiovascular pathology, was effective in the management of acute mania. ${ }^{38}$ However, the review suggests that this might be due to poor blood-brain barrier permeability of verapamil. There are also 
concerns about cardiovascular side effects of using L-type calcium channel blockers in bipolar disorder.

Gabapentin and pregabalin cross the blood-brain barrier readily $^{39} 40$ and have limited side-effect profiles. ${ }^{8}$ Gabapentin has also been found to reduce peak high-voltage-activated $\left(\mathrm{Ca}_{\mathrm{v}} 1\right.$ and $\left.\mathrm{Ca}_{\mathrm{v}} 2\right) \mathrm{Ca}^{2+}$ channel currents in rat dorsal root ganglion cultured cells, after incubation with the drug for 3 days. ${ }^{41}$ The same study found that gabapentin reduced cell-surface expression of $\mathrm{Ca}_{\mathrm{v}} 2$ channels in xenopus oocytes, although Hoppa et at $t^{42}$ found no evidence of reduced $\mathrm{Ca}_{\mathrm{v}} 2$ channel current or expression in rat hippocampal cells under the same conditions of gabapentin administration. The results from the study by Hendrich et al ${ }^{41}$ suggest that gabapentin (and pregabalin, as it binds to the same site on the $\alpha 2 \delta$ subunit of the calcium channel) may be effective at inhibiting calcium channel function and therefore may be useful in the management of bipolar disorder. The CACNA1C gene is also implicated in sleep/wake regulation. Genome-wide association studies have found CACNA1C mutations are associated with sleep latency and quality ${ }^{43}$ as well as narcolepsy. ${ }^{44}$ Additionally, CACNA1C knockout mice have been shown to have altered REM sleep. ${ }^{45}$ This link between calcium channels and sleep quality suggests that gabapentin and pregabalin may influence sleep patterns. Further evidence that this may be the case is that noradrenergic neurons in the locus coeruleus of the human brain fire at different rates depending on sleep state and are therefore implicated in regulation of sleep/wake states. ${ }^{46}$ Gabapentin and pregabalin have been shown to decrease GABA levels, thereby increasing glutamate and promoting descending noradrenergic inhibition in rat's locus coeruleus. ${ }^{47}{ }^{48}$ Sleep disturbance is often comorbid with bipolar disorder ${ }^{49}$ and increased firing of noradrenergic neutrons in the locus coeruleus has also been associated with stress, fear and anxiety. ${ }^{50}$ Therefore, the actions of gabapentin and pregabalin in the locus coeruleus may be responsible for their anxiolytic effect. Some of the most effective drugs for acute anxiety are the benzodiazepines, which work by increasing the action of GABA at $\mathrm{GABA}_{\mathrm{A}}$ receptors. ${ }^{51}$ However, the work of Yoshizumi et $a t^{47}$ and Suto et $a t^{48}$ suggest that gabapentin reduces presynaptic GABA release in rat's locus coeruleus. Hellsten et a $\hat{D}^{0}$ found different expression of $\mathrm{GABA}_{\mathrm{A}}$ receptors in rodents compared with humans and suggested that the human locus coeruleus may mediate anxiolytic and sedative effects of benzodiazepines. Despite uncertainty in the neurobiological justification for possible anxiolytic efficacy of gabapentin and pregabalin, the latter is licenced for generalised anxiety disorder and gabapentin has been used to treat anxiety disorders for many years.

In summary, gabapentin has been extensively prescribed off-label for psychiatric indications, including bipolar disorder and anxiety, with little established evidence of efficacy. Gabapentin and pregabalin, a very similar drug with the same mechanism of action, bind to a subunit of voltage-dependent calcium channels. These calcium channels are found in the human brain and are implicated in the aetiopathogenesis of bipolar disorder, anxiety and insomnia. Given the neurobiological argument described above and the common clinical coexistence of bipolar disorder, anxiety and insomnia, this systematic review aims to collect and critically appraise all the available evidence about efficacy and tolerability of gabapentin and pregabalin in the treatment of bipolar disorder, insomnia and anxiety.

\section{OBJECTIVES}

1a. To assess the efficacy of gabapentin and pregabalin in bipolar disorder in:

A attenuating acute manic/mixed episodes;

$\mathrm{B}$ attenuating acute depressive episodes;

C preventing relapse of any mood episodes.

1b. To assess the efficacy of gabapentin and pregabalin in the acute and long-term management of anxiety disorders.

1c. To assess the efficacy of gabapentin and pregabalin in the acute and long-term management of insomnia.

2. To assess the acceptability and tolerability of gabapentin and pregabalin in comparison with placebo or active treatment when used in the acute or long-term treatment of bipolar disorder, insomnia and anxiety.

\section{METHODS}

\section{Types of studies}

To assess efficacy and acceptability, only double-blind, RCTs will be included. For consideration of adverse effects (tolerability), single-blind or open-label RCTs and non-randomised evidence will also be summarised. For trials that have a crossover design, only results from the first period prior to crossover will be considered. Cluster randomised trials will be excluded. In bipolar disorder, acute treatment is the intervention given to patients with an acute manic, depressed or mixed episode (usually 3 weeks in manic and mixed episodes ${ }^{15}$ and 8 weeks for depression) $;{ }^{52}$ in contrast, long-term treatment is a treatment which had been instituted specifically or mainly to prevent further episodes of illness (either manic, mixed or depressive). ${ }^{53}$ In this review, we defined long-term treatment for bipolar disorder as treatment with a minimum duration of at least 3 months ( $>12$ weeks). ${ }^{54} 55$ For acute treatment of insomnia, the follow-up is usually $<1$ month, between 1 and 3 months for subchronic insomnia and more than 3 months for persistent insomnia. ${ }^{56}$ The overall aim of treatment for anxiety is to improve-and ideally achieve complete relief fromsymptoms and to prevent their recurrence; following an initial acute phase, treatment usually needs to be continued on a long-term basis, due to the chronic nature of the disease. ${ }^{57}$ For trials about anxiety, we will consider the authors' definition of acute and long-term management as reported in the original study. 


\section{Types of participants}

Patients of any age, of both sexes, any ethnicity, based in any clinical setting will be included.

\section{Bipolar disorder}

Only studies adopting standardised diagnostic criteria from the International Classification of Diseases (ICD) or the Diagnostic and Statistical Manual of Mental Disorders (DSM) to define patients suffering from bipolar disorder will be included. We will exclude studies which define bipolar disorder as scoring above a certain cut-off on a screening questionnaire. All subtypes of bipolar disorder (rapid cycling, type I, type II, not otherwise specified) will be included. No restrictions on clinician setting (eg, primary or secondary care) will be applied. Patients who have a concurrent primary diagnosis of an Axis I disorder or a serious concomitant medical illness will be excluded, with the exception of patients with comorbid anxiety and insomnia who will be analysed separately in a sensitivity analysis.

\section{Anxiety}

Studies relating to any anxiety disorder defined in the ICD or DSM will be included. These include, but are not limited to, panic disorder, generalised anxiety disorder and phobic anxiety disorders. Additionally, we will include studies which investigate anxiety in a population with no diagnosis of anxiety but who are observed during a period of stress, for example, preoperative anxiety. Patients who have a concurrent primary diagnosis of an Axis I disorder or a serious concomitant medical illness will be excluded, with the exception of patients with comorbid bipolar disorder and insomnia who will be analysed separately in a sensitivity analysis.

\section{Insomnia}

Any studies relating to sleep or insomnia will be included. This includes studies which assess sleep quality and patterns in normal persons as well as those which consider a population of patients with a defined sleep disorder. Patients who have a concurrent primary diagnosis of an Axis I disorder will be included, but patients with other serious concomitant medical illness will be excluded.

\section{Types of intervention}

\section{Experimental interventions}

Gabapentin or pregabalin in any dose, frequency, route of delivery or setting will be included. Trials in which gabapentin or pregabalin therapy is 'added-on' to preexisting treatments (eg, lithium) will be included if the pre-existing treatments are evenly distributed in the experimental and comparator intervention arms of the study. Trials which allow rescue medications (eg, shortterm use of hypnotics) will be included as long as these medications are equally distributed among the randomised intervention and comparator arms. Sensitivity analyses will then be performed to investigate if cotreatment or rescue medications are responsible for altering the efficacy of gabapentin or pregabalin in treating bipolar disorder, insomnia or anxiety.

\section{Comparator interventions}

Placebo or any other active pharmacological treatment (any preparation, dose, frequency, route of delivery or setting).

\section{Types of outcome measures}

\section{Primary outcomes}

1a Efficacy of gabapentin or pregabalin in the acute treatment of bipolar disorder, is as follows:

A. Number of hospital admissions during the study period;

B. Length of hospital admission;

C. Changes on validated manic or depressive symptom rating scales from baseline;

D. Changes on validated psychotic symptom rating scales from baseline;

E Response to treatment (ie, at least $50 \%$ improvement on any validated rating scale);

F Time to cessation of additional treatment for manic/depressive symptoms.

1b Efficacy of gabapentin or pregabalin in the longterm treatment of bipolar disorder:

A. Time to recurrence of any mood episodes;

B. Number of recurrences of any mood episodes during the trial period;

C. Number of recurrences of manic/mixed/ depressive episodes during the trial period;

D. Recurrence will be defined either as (i) study withdrawal due to recurrence of any mood episode, (ii) admission to hospital (time to next admission and number of admissions during trial period) or (iii) institution of additional treatment for any mood episode and time to institution.

2. Efficacy of gabapentin or pregabalin in the acute and long-term treatment of anxiety is as follows:

A. Change on validated and standardised anxiety rating scales.

3. Efficacy of gabapentin or pregabalin in the acute and long-term treatment of insomnia is as follows:

A. Objectively measured or self-reported sleep time;

B. Self-reported sleep quality;

C. Sleep onset latency.

\section{Secondary outcomes}

1. Acceptability of gabapentin or pregabalin in the acute and long-term treatment of bipolar disorder, anxiety and insomnia is measured as follows:

A. Participants dropping out of the treatment due to any cause;

B. Participants dropping out of the treatment due to adverse events;

C. Participants dropping out of the treatment due to inefficacy. 
2. Tolerability (adverse events) of gabapentin or pregabalin in the acute and long-term treatment of bipolar disorder, anxiety and insomnia is measured as follows:

A. Participants experiencing at least one troublesome side effect of any nature;

B. Participants experiencing each of the following specific side effects (BNF, 2016):
I. Amnesia
II. Anxiety
III. Convulsion
IV. Depression
V. Dizziness
VI. Drowsiness
VII. Emotional lability
VIII. Euphoria
IX. Insomnia
X. Leucopoenia
XI. Movement disorder
XII. Vertigo
XIII. Visual disturbance
XIV. Weight gain

In order not to miss any relatively rare or unexpected yet important adverse events, in the data extraction phase, we will collect all side-effect data reported in the included studies and discuss ways to summarise them post hoc.

\section{Search methods for identification of studies \\ Electronic searches}

Searches for published studies will be undertaken in the following electronic bibliographic databases: CENTRAL, EMBASE, MEDLINE, MEDLINE In-Process and PsycINFO. The following phrase will be used: ((( ('bipolar disorder' OR 'cyclothymic disorder') OR 'sleep initiation and maintenance disorders' OR ('anxiety' OR 'anxiety disorders' OR 'agoraphobia' OR 'anxiety, separation' OR 'combat disorders' OR 'neurotic disorders' OR 'obsessive-compulsive disorder' OR 'panic disorder' OR 'phobic disorders' OR 'stress disorders, traumatic' OR 'stress disorders, post-traumatic' OR 'psychological trauma' OR 'stress disorders, traumatic, acute')) AND ('gamma-aminobutyric acid' OR 'pregabalin')) combined with terms for randomised controlled trials (efficacy) OR adverse effects (tolerability))*

*Subject (MeSH) entries sourced from MEDLINE.

\section{Searching other resources}

\section{Research registers}

We will search ClinicalTrials.gov and the WHO's trials portal (ICTRP) to identify unpublished or ongoing studies. There will be no date, language or publication status restrictions to the searches.

\section{Grey literature}

We will conduct complementary searches of the following drug approval agencies for additional published and unpublished data: The European Medicines Agency
(EU), the Food and Drug Administration (USA), the Medicines and Healthcare products Regulatory Agency (UK), the Medicines Evaluation Board (the Netherlands), the Medical Products Agency (Sweden), the Pharmaceuticals and Medical Devices Agency (Japan) and the Therapeutic Goods Administration (Australia).

\section{Reference lists}

We will check the reference lists of all included studies, relevant papers and previous systematic reviews for identification of additional studies that may be missed by the electronic database searches. We will undertake a cited reference search of the included studies in the Web of Science.

\section{Data collection and analysis \\ Selection of studies}

Three review authors (AF, AA, LZA) will independently check the titles and abstracts of all of the references generated by the search strategies to decide if they meet the inclusion criteria. All references potentially eligible for inclusion certified by either of the three reviewers will be added to the preliminary list, and their full-text articles will be retrieved. The three authors will then assess all the corresponding full-text articles to see if they still meet the inclusion criteria. If the authors disagree, the final decision will be made by consensus with the involvement of another member of the team.

\section{Data extraction and management}

KTH, AA and LZA will independently extract data from the included studies. Any disagreement will be discussed, and decisions documented. If necessary, we will contact authors of studies for clarification and original data not included in published papers. The following data will be extracted from all studies meeting the inclusion criteria:

- Study characteristics (blinding, randomisation, sponsorship, crossover/parallel group design),

- Participant characteristics (age, sex, ethnicity, study setting, primary diagnosis according to DSM or ICD classification, comorbidity, severity, treatment history for the index episode),

- Intervention details (intervention treatment, comparator treatment, dosage, frequency of administration, route of administration, duration of therapy, cointerventions),

- Outcome measures of interest in terms of efficacy, tolerability and adverse events.

\section{Assessment of risk of bias in included studies}

Three authors (AF, AA, LZA) will independently assess trial quality using the Cochrane risk of bias tool. ${ }^{58}$ The following factors will be assessed: (1) sequence generation, (2) allocation concealment, (3) blinding, (4) incomplete outcome data, (5) selective reporting and (6) other potential sources of bias. Each item will be 
rated as high, low or unclear risk of bias, and a justification from the study report will be supplied to support the judgement as appropriate. If the authors disagree, the final decision will be made by consensus with another review author (AC).

\section{Measures of treatment effect \\ Dichotomous data}

For dichotomous, or event-like, data, the risk ratio (RR) will be calculated with its $95 \%$ CI. For statistically significant results, we will calculate the number needed to treat for an additional beneficial outcome and the number needed to treat for an additional harmful outcome, as the inverse of the risk difference.

\section{Continuous data}

For continuous data, mean differences (MDs) or standardised MDs (SMDs) will be calculated with 95\% CIs. MDs will be used when the same scale is used to measure an outcome; SMDs will be employed when different scales are used to measure the same outcome.

Continuous data on clinical outcomes often are not normally distributed, and skewed data will be presented descriptively. If papers report a mean and an SD, as well as an absolute minimum possible value for the outcome, we will divide the mean by the SD. If this value is $<2$, then we will conclude that some indication of skewness is present. If the value is $<1$ (ie, the $\mathrm{SD}$ is bigger than the mean), then skewness will almost certainly be present. If papers do not report the skewness and simply report means, SDs and sample sizes, these numbers will be used. As these data may not have been properly analysed and can be misleading, fresh analyses will be conducted with and without these studies. If the data are log-transformed for analysis, and the geometric means reported, skewness will be reduced. This is the recommended method for analysis of skewed data. ${ }^{58}$

\section{Studies with multiple treatment groups}

For a particular multiarm study, the intervention groups of relevance to a systematic review are all those that could be included in a pairwise comparison of intervention groups that, if investigated alone, would meet the criteria for inclusion of studies in the review. Each meta-analysis addresses only a single pairwise comparison, so we will first consider whether a study of each possible pairwise comparison of interventions in the study is eligible for the meta-analysis. Then, several possible approaches to including a study with multiple intervention groups could be used in a particular meta-analysis. ${ }^{58}$ For binary outcomes, if possible, we will combine all relevant experimental intervention groups of the study into a single experimental group, and combine all relevant control intervention groups into a single control group. For continuous outcomes, we will combine means and SDs using methods described in Cochrane Handbook. ${ }^{58}$

\section{Dealing with missing data}

Binary outcomes will be calculated on a strict intention-to-treat (ITT) basis: dropouts will be included in this analysis. When data are missing and the method of last observation carried forward (LOCF) has been used to do an ITT analysis, then the LOCF data will be used. ${ }^{59}$ When SDs are missing, we will present data descriptively. When SDs are not reported, we will ask authors to supply the data. When only the SE or tstatistics or the $p$ value is reported, we will calculate SDs in accordance with Altman and Bland. ${ }^{60}$

\section{Data synthesis}

We will conduct a random-effects meta-analysis, whenever possible (ideally, we plan to perform separate meta-analyses for acute and long-term treatment in each disorder) ${ }^{61}$ We understand that there is no closed argument for preference for the use of fixed or random-effects models. The random-effects method incorporates an assumption that different studies are estimating different, yet related, intervention effects, and takes into account differences between studies even if there is no statistically significant heterogeneity. The disadvantage of the random-effects model, though, is that it puts added weight onto small studies, which often are the most biased ones. Depending on the direction of effect, these studies can either inflate or deflate the effect size.

\section{Assessment of heterogeneity}

Heterogeneity between studies will be investigated by the $\mathrm{I}^{2}$ statistic $^{62}\left(\mathrm{I}^{2} \geq 50 \%\right.$ will be considered indicative of significant heterogeneity) and by visual inspection of the forest plots. Given that the value of $\mathrm{I}^{2}$ depends on the sample size of the included studies, the magnitude and direction of effects and the strength of evidence for heterogeneity, we will use arbitrary threshold to perform a preliminary evaluation. If the $\mathrm{I}^{2}$ value is below $50 \%$ but the direction and magnitude of treatment effects are suggestive of important heterogeneity, we will investigate the potential sources of heterogeneity.

\section{Assessment of reporting biases}

Data from included studies will be entered into a funnel plot (trial effect against trial variance) for investigation of small-study effects. ${ }^{63}$ We plan to use the tests for funnel plot asymmetry only if at least 10 studies are included in the meta-analysis. ${ }^{58}$ Funnel plot asymmetry may be noted for many possible reasons, so if evidence of small-study effects are identified, all possible reasons for funnel plot asymmetry, including publication bias, will be investigated. ${ }^{64}$

\section{Subgroup and sensitivity analyses}

No subgroup analysis will be undertaken. In contrast, we will carry out the following sensitivity analyses:

- excluding trials in which gabapentin or pregabalin were used as 'add-on' therapy to another treatment 
to determine if coprescription may affect the efficacy of gabapentin or pregabalin;

- excluding trials involving patients with any combination of psychiatric comorbidity;

- excluding trials which allow rescue medications (eg, short-term use of hypnotics);

- as all data will be synthesised using a random-effects model, we will also synthesise data for the primary outcome using a fixed-effects model to evaluate whether the greater weights assigned to larger trials with greater event rates can alter the significance of the results compared with the more evenly distributed weights in the random-effects model.

\section{Summary of findings table}

We will use the Grades of Recommendation, Assessment, Development and Evaluation approach to assess the quality of the supporting evidence behind each estimate of treatment effect. We will use risk of bias, imprecision, inconsistency, indirectness and publication bias, to rate the overall evidence. ${ }^{65}$ We will present key findings of the review in a 'summary of findings' table. This will include a summary of the amount of data, the magnitude of the effect size and the overall quality of the evidence for the primary outcomes.

\section{Dissemination}

We will publish findings from this systematic review in a peer-reviewed scientific journal and data set will be made freely available. The completed review will be disseminated electronically, in print and on social media, where appropriate.

This protocol has been registered on PROSPERO (CRD42016041802).

Twitter Follow Andrea Cipriani @And_Cipriani and John Geddes @0xPsychiatry

Contributors AC, PJH and JRG devised the study. KTH and AC drafted the protocol. AF, AA, KTH, LZA and AC will select the references and will carry out most of the data collection. AC will analyse the data and draft the results and discussion sections. SS designed and conducted the search strategy for published and unpublished studies. AF, AA, LZA, PJH, SS and JRG revised the protocol, assisted with study design and will help with the final manuscript. AC is the guarantor of the review.

Funding The authors' bipolar disorder research is supported by a Wellcome Trust Strategic Award (CONBRIO: Collaborative Network for Bipolar Research to Improve Outcomes) and by the NIHR Oxford Health Biomedical Research Centre. AC is supported by the NIHR Oxford cognitive health Clinical Research Facility. JG is an NIHR Senior Investigator. This review has been conducted under the auspices of the Oxford Cognitive Health and Neuroscience Clinical Trials Unit in which AC leads the research synthesis programme.

Disclaimer The funder has no role in developing the protocol. The views expressed here are those of the authors and not necessarily those of the funders, the National Health Service, the NIHR or the Department of Health.

Competing interests In the past 2 years: AC has served as an expert witness for a patent litigation case about quetiapine extended release. PJH has served as an expert witness on patent litigation involving drugs used to treat bipolar disorder. The other authors report no conflicts of interest.

Ethics approval Not required.

Provenance and peer review Not commissioned; externally peer reviewed.
Data sharing statement We will publish findings from this systematic review in a peer-reviewed scientific journal, and data set will be made freely available.

Open Access This is an Open Access article distributed in accordance with the terms of the Creative Commons Attribution (CC BY 4.0) license, which permits others to distribute, remix, adapt and build upon this work, for commercial use, provided the original work is properly cited. See: http:// creativecommons.org/licenses/by/4.0/

\section{REFERENCES}

1. Mack A. Examination of the evidence for off-label use of gabapentin. J Manag Care Pharm 2003;9:559-68.

2. Rosner H, Rubin L, Kestenbaum A. Gabapentin adjunctive therapy in neuropathic pain states. Clin J Pain 1996;12:56-8.

3. Field MJ, Oles RJ, Lewis AS, et al. Gabapentin (neurontin) and S-(+)-3-isobutylgaba represent a novel class of selective antihyperalgesic agents. Br J Pharmacol 1997;121:1513-22.

4. Gillin S, Sorkin LS. Gabapentin reverses the allodynia produced by the administration of anti-GD2 ganglioside, an immunotherapeutic drug. Anesth Analg 1998;86:111-16.

5. Silverman RB. From basic science to blockbuster drug: the discovery of Lyrica. Angew Chem Int Ed Engl 2008;47:3500-4.

6. Food and Drug Administration. Drug and New Biologic Approvals in 2004. Document. http://www.fda.gov/drugs/development approvalprocess/howdrugsaredevelopedandapproved/ drugandbiologicapprovalreports/ucm081677.htm (accessed on 14 Apr 2016).

7. Food and Drug Administration. Efficacy Supplement Approvals in 2005. Document. http://www.fda.gov/drugs/ developmentapprovalprocess/howdrugsaredevelopedandapproved/ drugandbiologicapprovalreports/ucm081892.htm (accessed $14 \mathrm{Apr}$ 2016).

8. British National Formulary. Joint Formulary Committee. British National Formulary (online). http://www.evidence.nhs.uk/formulary/ bnf/current/4-central-nervous-system/48-antiepileptic-drugs/ 481-control-of-the-epilepsies/gabapentin-and-pregabalin (accessed 11 Jul 2016).

9. González-Bueno J, Calvo-Cidoncha E, Desongles-Corrales T, et al. DI-024 off-label use of gabapentin and pregabalin in a tertiary hospital. Eur J Hosp Pharm 2015;22:A87-8.

10. Hamer AM, Haxby DG, McFarland BH, et al. Gabapentin use in a managed Medicaid population. J Manag Care Pharm 2002;8:266-71.

11. Berlin RK, Butler PM, Perloff MD. Gabapentin therapy in psychiatric disorders: a systematic review. Prim Care Companion CNS Disord 2015;17:5.

12. Pande AC, Crockatt JG, Janney CA, et al. Gabapentin in bipolar disorder: a placebo-controlled trial of adjunctive therapy. Gabapentin Bipolar Disorder Study Group. Bipolar Disord 2000;2:249-55.

13. Frye MA, Ketter TA, Kimbrell TA, et al. A placebo controlled study of lamotrigine and gabapentin monotherapy in refractory mood disorders. J Clin Psychopharmacol 2000;20:607-14.

14. Obrocea GV, Dunn RM, Frye MA, et al. Clinical predictors of response to lamotrigine and gabapentin monotherapy in refractory affective disorders. Biol Psychiatry 2002;51:253-60.

15. Cipriani A, Barbui C, Salanti G, et al. Comparative efficacy and acceptability of antimanic drugs in acute mania: a multiple-treatments meta-analysis. Lancet 2011;378:1306-15.

16. Vieta E, Goikolea JM, Martínez-Arán A, et al. A double-blind, randomized, placebo-controlled, prophylaxis study of adjunctive gabapentin for bipolar disorder. J Clin Psychiatry 2006;67:473-7.

17. Pande AC, Davidson JR, Jefferson JW, et al. Treatment of social phobia with gabapentin: a placebo controlled study. J Clin Psychopharmacol 1999;19:341-8.

18. Food and Drug Administration. Drug maker to pay $\$ 430$ million in fines, civil damages. FDA Consum 2004;38:36-7.

19. Baldwin DS, Ajel K. Role of pregabalin in the treatment of generalized anxiety disorder. Neuropsychiatr Dis Treat 2007;3:185

20. Schaffer LC, Schaffer CB, Miller AR, et al. An open trial of pregabalin as an acute and maintenance adjunctive treatment for outpatients with treatment resistant bipolar disorder. J Affect Disord 2013;147:407-10.

21. Satzinger G. Antiepileptics from $\gamma$-aminobutyric acid. Drug Res 1994;44:261-6.

22. Cheng JK, Chiou LC. Mechanisms of the antinociceptive action of gabapentin. J Pharmacol Sci 2006;100:471-86.

23. Gee NS, Brown JP, Dissanayake VU, et al. The novel anticonvulsant drug, gabapentin (Neurontin), binds to the subunit of a calcium channel. J Biol Chem 1996;271:5768-76. 
24. Bian F, Li Z, Offord J. Calcium channel alpha2-delta type 1 sub-unit is the major binding protein for pregabalin in neocortex hippocampus, amygdala, and spinal cord: an ex vivo autoradiographic study in alpha2 delta type 1 genetically modified mice. Brain Res 2006;1075:68-80.

25. Field MJ, Cox PJ, Stott E, et al. Identification of the $\alpha 2-\delta-1$ subunit of voltage-dependent calcium channels as a molecular target for pain mediating the analgesic actions of pregabalin. Proc Natl Acad Sci USA 2006;103:17537-42.

26. Lotarski S, Hain $\mathrm{H}$, Peterson J, et al. Anticonvulsant activity of pregabalin in the maximal electroshock-induced seizure assay in $\alpha_{2} \delta_{1}(\mathrm{R} 217 \mathrm{~A})$ and $\alpha_{2} \delta_{2}$ (R279A) mouse mutants. Epilepsy Res 2014;108:833-42.

27. Catterall WA. Structure and regulation of voltage-gated $\mathrm{Ca} 2+$ channels. Annu Rev Cell Dev Biol 2000;16:521-55.

28. Dolphin AC. Calcium channel diversity: multiple roles of calcium channel subunits. Curr Opin Neurobiol 2009;19:237-44.

29. Arikkath J, Campbell KP. Auxiliary subunits: essential components of the voltage-gated calcium channel complex. Curr Opin Neurobiol 2003;13:298-307.

30. Ertel EA, Campbell KP, Harpold MM, et al. Nomenclature of voltage-gated calcium channels. Neuron 2000;25:533-5

31. Ferreira MA, O'Donovan MC, Meng YA, et al. Collaborative genome-wide association analysis supports a role for ANK3 and CACNA1C in bipolar disorder. Nat Genet 2008;40:1056-8.

32. Sklar P, Smoller JW, Fan J, et al. Whole-genome association study of bipolar disorder. Mol Psychiatry 2008;13:558-69.

33. Bigos KL, Mattay VS, Callicott JH, et al. Genetic variation in CACNA1C affects brain circuitries related to mental illness. Arch Gen Psychiatry 2010;67:939-45.

34. Yoshimizu T, Pan JQ, Mungenast AE, et al. Functional implications of a psychiatric risk variant within CACNA1C in induced human neurons. Mol Psychiatry 2015;20:162-9.

35. Yamawaki S Kagaya A, Tawara Y, et al. Intracellular calcium signaling systems in the pathophysiology of affective disorders. Life Sci 1998:62:1665-70.

36. Simpson PB, Russell JT. Role of mitochondrial $\mathrm{Ca}^{2+}$ regulation in neuronal and glial cell signaling. Brain Res Brain Res Rev 1998;26:72-81.

37. Stork C, Renshaw PF. Mitochondrial dysfunction in bipolar disorder: evidence from magnetic resonance spectroscopy research. $\mathrm{Mol}$ Psychiatry 2005;10:900-19.

38. Cipriani A, Saunders K, Attenburrow MJ, et al. A systematic review of calcium channel antagonists in bipolar disorder and some considerations for their future development. Mol Psychiatry 2016;21:1324-32.

39. McLean MJ. Clinical pharmacokinetics of gabapentin. Neurology 1994:44(6 Suppl 5):S17-22; discussion S31-2.

40. Ben-Menachem E. Pregabalin pharmacology and its relevance to clinical practice. Epilepsia 2004:45(Suppl 6):13-18

41. Hendrich J, Van Minh AT, Heblich F, et al. Pharmacological disruption of calcium channel trafficking by the $\alpha 2 \delta$ ligand gabapentin. Proc Natl Acad Sci USA 2008;105:3628-33.

42. Hoppa MB, Lana B, Margas W, et al. $\alpha 2 \delta$ expression sets presynaptic calcium channel abundance and release probability. Nature 2012:486:122-5.

43. Byrne EM, Gehrman PR, Medland SE, et al. A genome-wide association study of sleep habits and insomnia. Am J Med Genet B Neuropsychiatr Genet 2013;162B:439-51.

44. Shimada M, Miyagawa T, Kawashima M, et al. An approach based on a genome-wide association study reveals candidate loci for narcolepsy. Hum Genet 2010;128:433-41.
45. Kumar D, Dedic N, Flachskamm C, et al. Cacna1c (Cav1. 2) modulates electroencephalographic rhythm and rapid eye movement sleep recovery. Sleep 2015;38:1371-80.

46. Saper CB, Chou TC, Scammell TE. The sleep switch: hypothalamic control of sleep and wakefulness. Trends Neurosci 2001;24:726-31.

47. Yoshizumi M, Parker RA, Eisenach JC et al Gabapentin inhibits $\gamma$-amino butyric acid release in the locus coeruleus but not in the spinal dorsal horn after peripheral nerve injury in rats. Anesthesiology 2012;116:1347-53.

48. Suto T, Severino AL, Eisenach JC, et al. Gabapentin increases extracellular glutamatergic level in the locus coeruleus via astroglial glutamate transporter-dependent mechanisms. Neuropharmacology 2014;81:95-100.

49. Plante DT, Winkelman JW. Sleep disturbance in bipolar disorder: therapeutic implications. Am J Psychiatry 2008;165:830-43.

50. Hellsten KS, Sinkkonen ST, Hyde TM, et al. Human locus coeruleus neurons express the GABA A receptor $\gamma 2$ subunit gene and produce benzodiazepine binding. Neurosci Lett 2010;477:77-81.

51. Nemeroff CB. The role of GABA in the pathophysiology and treatment of anxiety disorders. Psychopharmacol Bull 2002;37:133-46.

52. Harrison PJ, Cipriani A, Harmer CJ, et al. Innovative approaches to bipolar disorder and its treatment. Ann N Y Acad Sci 2016;1366:76-89.

53. Cipriani A, Barbui C, Rendell J, et al. Clinical and regulatory implications of active run-in phases in long-term studies for bipolar disorder. Acta Psychiatr Scand 2014;129:328-42.

54. Miura T, Noma H, Furukawa TA, et al. Comparative efficacy and tolerability of pharmacological treatments in the maintenance treatment of bipolar disorder: a systematic review and network meta-analysis. Lancet Psychiatry 2014;1:351-9.

55. Cipriani A, Hawton $\mathrm{K}$, Stockton $\mathrm{S}$, et al. Lithium in the prevention of suicide in mood disorders: updated systematic review and meta-analysis. BMJ 2013;346:f3646.

56. Morin CM, Benca R. Chronic insomnia. Lancet 2012;379:1129-41.

57. National Collaborating Centre for Mental Health. Generalised anxiety disorder and panic disorder in adults: management. https://www. nice.org.uk/guidance/cg113 (accessed 5 Jan 2017).

58. Higgins JPT, Green S, eds. Cochrane handbook for systematic reviews of interventions, Version 5.1.0 [updated March 2011]. The Cochrane Collaboration, 2011. http://handbook.cochrane.org

59. Mavridis D, Chaimani A, Efthimiou O, et al. Addressing missing outcome data in meta-analysis. Evid Based Mental Health 2014;17:85-9.

60. Altman DG, Bland JM. Detecting skewness from summary information. BMJ 1996;313:1200.

61. Nikolakopoulou A, Mavridis D, Salanti G. Demystifying fixed and random effects meta-analysis. Evid Based Mental Health 2014;17:53-7.

62. Higgins JP, Thompson SG, Deeks JJ, et al. Measuring inconsistency in meta-analyses. BMJ 2003;327:557-60.

63. Sterne JA, Gavaghan D, Egger M. Publication and related bias in meta-analysis: power of statistical tests and prevalence in the literature. J Clin Epidemiol 2000:53:1119-29.

64. Mavridis D, Salanti G. Exploring and accounting for publication bias in mental health: a brief overview of methods. Evid Based Mental Health 2014;17:11-15.

65. Balshem $\mathrm{H}$, Helfand M, Schunemann $\mathrm{HJ}$, et al. GRADE guidelines: 3 . Rating the quality of evidence. J Clin Epidemiol 2011;64:401-6. 